\title{
COHOMOlOGY THEORIES OF Hopf Bimodules AND CUP-PRODUCT.
}

\author{
Rachel Taillefer*
}

\begin{abstract}
Given a Hopf algebra $A$, there exist various cohomology theories for the category of Hopf bimodules over $A$, introduced by M. Gerstenhaber and S.D. Schack, and by C. Ospel. We prove, when $A$ is finite dimensional, that they are all equal to the Ext functor on the module category of an associative algebra associated to $A$, described by $\mathrm{C}$. Cibils and M. Rosso. We also give an expression for a cup-product in the cohomology defined by C. Ospel, and prove that it corresponds to the Yoneda product of extensions.
\end{abstract}

2000 Mathematics Subject Classification: 16E30, 16E40, 16S99, 16W30, 57T05.

Keywords: Cohomology, Hopf algebras.

\section{Introduction}

Let $A$ be a finite dimensional Hopf algebra over a field $k$, and let $M$ and $N$ be Hopf bimodules over $A$. We shall consider various cohomology theories associated to these objects. In the first place, M. Gerstenhaber and S.D. Schack have defined, in GS1, a cohomology $H_{b}^{*}(A, A)$ for $A$. It arose when studying the deformations of $A$ (however, this cohomology does not characterize the deformations of $A$ in the category of Hopf algebras, but in a larger class of objects which includes Hopf algebras and Drinfel'd algebras). Next, in [GS2], M. Gerstenhaber and S.D. Schack extended this cohomology to a cohomology theory $H_{G S}^{*}(M, N)$ of $M$ into $N$. Lastly, C. Ospel defined in his thesis O a cohomology $H_{A 4}^{*}(M, A)$ of $M$ with coefficients in $A$, introducing a double complex which involves both Hochschild cohomology complexes and Cartier cohomology complexes $([\mathrm{G}])$.

In this paper, we will show that these cohomologies are all the same. To this end, we will introduce an associative algebra $X$, defined by C. Cibils and M. Rosso in [CR], and view all Hopf bimodules as left $X$-modules. The above cohomologies will then be isomorphic to the space of extensions $\operatorname{Ext}_{X}^{*}(M, N)$.

We will further give expressions of the Yoneda product of extensions in $H_{A 4}^{*}(M, N)$ (which generalizes $\left.H_{A 4}^{*}(M, A)\right)$ and $H_{b}^{*}(A, A)$, which do not involve $X$.

There appears to be a natural definition for the cyclic cohomology of a finite dimensional Hopf algebra, that is the cyclic cohomology of the associative algebra $X$. On the other hand, in [CM], A. Connes and H. Moscovici have defined a cyclic module associated to a

${ }^{*}$ Laboratoire G.T.A., UPRES A 5030, Département de Mathématiques CC 051, Université Montpellier II, 34095 Montpellier Cedex 5, France. E-mail: taillefr@math.univ-montp2.fr 
Hopf algebra endowed with a modular pair in involution. These theories differ; moreover, although the cyclic cohomology of $X$ takes equally into account all the structures of $A$, the cyclic cohomology of $A$ defined by A. Connes and H. Moscovici gives a greater weight to the coalgebra structure of $A$. However, it would be interesting to see whether there is a connection between them.

This paper is organized as follows: we will first describe the algebra $X$. Then we will describe M. Gerstenhaber and S.D. Schack's cohomologies $H_{G S}^{*}(M, N)$ and $H_{b}^{*}(A, A)$, explain why the first extends the second, and prove that $H_{G S}^{*}(M, N)$ is isomorphic to $\operatorname{Ext}_{X}^{*}(M, N)$. Next, we shall do the same with a generalization of the cohomology defined by $\mathrm{C}$. Ospel. Finally, we shall describe a cup-product on this last cohomology and on $H_{b}^{*}(A, A)$, and prove that it corresponds to the Yoneda product of extensions.

We shall need some notation: all tensor products will be taken over the base field $k$. The structure maps of the Hopf algebra $A$ are denoted in the standard way $\mu, \Delta, \eta, \varepsilon$ and $S$. We will use Sweedler's notations for the comultiplications:

$$
\begin{aligned}
& \Delta(x)=\sum x^{(1)} \otimes x^{(2)} \text { if } x \in A, \\
& \mu^{*}(l)=\sum l_{(1)} \otimes l_{(2)} \text { if } l \in A^{*},
\end{aligned}
$$

and the comodule structures:

$$
\begin{aligned}
& M \longrightarrow A \otimes M \quad \text { and } M \longrightarrow \quad \longrightarrow \otimes A \\
& m \mapsto \sum m_{(-1)} \otimes m_{(0)} \quad m \mapsto \sum m_{(0)} \otimes m_{(1)} .
\end{aligned}
$$

Let us introduce, for any integer $u \geq-1$, the map:

$$
\begin{aligned}
\Delta^{(u)}: A & \longrightarrow A^{\otimes u+1} \\
a & \mapsto \begin{cases}\varepsilon(a) & \text { if } u=-1, \\
a & \text { if } u=0, \\
\left(\Delta \otimes 1^{\otimes u-1}\right) \ldots(\Delta \otimes 1) \Delta(a) & \text { otherwise. }\end{cases}
\end{aligned}
$$

The results in this paper will form part of my $\mathrm{PhD}$ thesis. I am happy to thank my advisor Claude Cibils and Jean-Michel Oudom for their help and comments.

\section{The algebra $X$.}

Let $A$ be a finite dimensional Hopf algebra over a field $k$. In CR, C. Cibils and M. Rosso have introduced an associative $k$-algebra $X$, in order to identify Hopf bimodules over $A$ with left modules over $X$ (just as bimodules over an associative algebra are identified with left modules over its enveloping algebra). This they do by viewing the right $A$-module structure of a Hopf bimodule as a left $A^{o p}$-module stucture, its left coaction as a left action of $A^{* o p}$, and its right coaction as a left action of $A^{*}$. To preserve the compatibilities between the structures, they endow $A^{*}$ and $A^{* o p}$ with $A$-bimodule structures involving the antipode. Thus they define the algebra $X$ as being the vector space $\left(A^{* o p} \otimes A^{*}\right) \underline{\otimes}\left(A \otimes A^{o p}\right)$ in which the first two and last two tensorands keep natural multiplication, and

$$
\begin{aligned}
& {[(1 \otimes 1) \underline{\otimes}(a \otimes b)][(l \otimes k) \underline{\otimes}(1 \otimes 1)]} \\
& \quad=\sum l_{(1)}\left(S a^{(1)}\right) k_{(1)}\left(S^{-1} a^{(3)}\right) l_{(3)}\left(S^{-1} b^{(1)}\right) k_{(3)}\left(S b^{(3)}\right)\left[\left(l_{(2)} \otimes k_{(2)}\right) \underline{\otimes}\left(a^{(2)} \otimes b^{(2)}\right)\right],
\end{aligned}
$$


and they prove the following:

Theorem 2.1 (CR theorem 3.1) There is a vector space-preserving equivalence of categories between the category of left modules over $X$ and the category of Hopf bimodules over $A$.

Remarks (i) If $M$ is a Hopf bimodule, its left $X$-module structure is given by:

$$
[(l \otimes k) \underline{\otimes}(a \otimes b)] . m=\sum l\left(a^{(1)} m_{(-1)} b^{(1)}\right) k\left(a^{(3)} m_{(1)} b^{(3)}\right)\left(a^{(2)} m_{(0)} b^{(2)}\right) .
$$

(ii) C. Cibils and M. Rosso have also proved that the algebra $X$ is isomorphic to the direct tensor product $\mathcal{H}(A) \otimes \mathcal{D}(A)^{o p}$, where $\mathcal{H}(A)$ is the Heisenberg double of $A$, and $\mathcal{D}(A)$ is the Drinfel'd double of $A$.

Henceforth, we will identify Hopf bimodules over $A$ with left $X$-modules, and, whenever $M$ and $N$ are Hopf bimodules, we shall consider the algebra $\operatorname{Ext}_{X}^{*}(M, N)$.

\section{Gerstenhaber and Schack's cohomology for Hopf bimodules}

This has been defined by M. Gerstenhaber and S.D. Schack in GS2]; we shall also use various notions introduced by S. Shnider and S. Sternberg in Sh-St].

\subsection{Bar resolution of a Hopf bimodule}

Let us first consider a bimodule $M$ over $A$. Recall that the bar complex $B_{\bullet}(M)$ of $M$ is the direct sum $\bigoplus_{q \geq-1} B_{q}(M)$, in which the $B_{q}(M)=A^{\otimes q+1} \otimes M \otimes A^{\otimes q+1}$ are bimodules via the standard actions (i.e. multiplication of the leftmost or rightmost tensorand), the differential $\partial_{q}: B_{q}(M) \rightarrow B_{q-1}(M)$ sends $a_{0} \otimes \ldots \otimes a_{q} \otimes m \otimes b_{q} \otimes \ldots \otimes b_{0}$ to

$$
\begin{aligned}
\sum_{i=0}^{q-1}(-1)^{i} a_{0} \otimes \ldots \otimes a_{i} a_{i+1} \otimes \ldots \otimes m \otimes & \ldots \otimes b_{i+1} b_{i} \otimes \ldots \otimes b_{0} \\
& +(-1)^{q} a_{0} \otimes \ldots \otimes a_{q-1} \otimes a_{q} m b_{q} \otimes b_{q-1} \otimes \ldots \otimes b_{0}
\end{aligned}
$$

for $q \geq 0$, and $\partial_{-1}=0$. This defines a complex of $A$-bimodules. To see that it is a resolution of $M$, let us introduce the homotopy

$$
\begin{aligned}
h_{q}: B_{q}(M) & \rightarrow B_{q+1}(M) \\
x & \mapsto 1 \otimes x \otimes 1 .
\end{aligned}
$$

It satisfies the equation $\partial_{q+1} h_{q}+h_{q-1} \partial_{q}=i d$, for all $q \geq-1$.

Assume now that $M$ is a Hopf bimodule over $A$. Let us endow the $B_{q}(M)$ with codiagonal coactions, that is

$$
\begin{aligned}
B_{q}(M) \rightarrow & A \otimes B_{q}(M) \\
\underline{a} \otimes m \otimes \underline{b} \mapsto & \sum a_{0}^{(1)} \ldots a_{q}^{(1)} m_{(-1)} b_{q}^{(1)} \ldots b_{0}^{(1)} \\
& \otimes a_{0}^{(2)} \otimes \ldots \otimes a_{q}^{(2)} \otimes m_{(0)} \otimes b_{q}^{(2)} \otimes \ldots \otimes b_{0}^{(2)},
\end{aligned}
$$

and similarly on the right. This makes $B_{\bullet}(M)$ into a complex of Hopf bimodules. The homotopy becomes a morphism of $A$-bicomodules, and $B_{\bullet}(M)$ is therefore a Hopf bimodule resolution of $M$ which splits as a sequence of $A$-bicomodules. 


\subsection{Cobar resolution of a Hopf bimodule}

Dually, let $N$ be a bicomodule over $A$. The cobar complex $C^{\bullet}(N)$ is equal to the direct sum $\bigoplus_{p \geq-1} C^{p}(N)$, where the vector spaces $C^{p}(N)=A^{\otimes p+1} \otimes N \otimes A^{\otimes p+1}$ are bicomodules via the standard coactions (that is comutiplication on the first or last tensorand), the differential $\partial^{p}: C^{p}(N) \rightarrow C^{p+1}(N)$ sends

$a_{0} \otimes \ldots \otimes a_{p} \otimes n \otimes b_{p} \otimes \ldots \otimes b_{0}$ to

$$
\begin{aligned}
\sum_{i=0}^{p}(-1)^{i} a_{0} \otimes \ldots \otimes \Delta a_{i} & \otimes \ldots \otimes n \otimes \ldots \otimes \Delta b_{i} \otimes \ldots \otimes b_{0} \\
& +(-1)^{p+1} \sum a_{0} \otimes \ldots \otimes a_{p} \otimes n_{(-1)} \otimes n_{(0)} \otimes n_{(1)} \otimes b_{p} \otimes \ldots \otimes b_{0},
\end{aligned}
$$

and $\partial^{-1}=0$.

There is a homotopy

$$
\begin{aligned}
h^{p}: C^{p}(N) & \rightarrow C^{p-1}(N) \\
\underline{a} \otimes n \otimes \underline{b} & \mapsto \varepsilon\left(a_{0}\right) \varepsilon\left(b_{0}\right) a_{1} \otimes \ldots \otimes a_{p} \otimes n \otimes b_{p} \otimes \ldots \otimes b_{1}
\end{aligned}
$$

which enables us to see that $C^{\bullet}(N)$ is a bicomodule resolution of $N$.

When $N$ is a Hopf bimodule, the $C^{p}(N)$ are endowed with diagonal actions, that is

$$
\begin{aligned}
A \otimes C^{p}(N) & \rightarrow C^{p}(N) \\
c \otimes \underline{a} \otimes n \otimes \underline{b} & \mapsto \sum c^{(1)} a_{0} \otimes \ldots \otimes c^{(p+1)} a_{p} \otimes c^{(p+2)} n \otimes \ldots \otimes c^{(2 p+3)} b_{0} \\
& =\Delta^{(2 p+2)}(c)\left(a_{0} \otimes \ldots \otimes a_{p} \otimes n \otimes b_{p} \otimes \ldots \otimes b_{0}\right),
\end{aligned}
$$

and similarly on the right, so that $C^{\bullet}(N)$ becomes a Hopf bimodule resolution of $N$ which splits as a sequence of $A$-bimodules.

\subsection{Gerstenhaber and Schack cohomology of Hopf bimodules}

Let $M$ and $N$ be two Hopf bimodules over $A$. As in [GS2], set

$$
C^{p q}(M, N)=\operatorname{Hom}_{A 4}\left(B_{q}(M), C^{p}(N)\right)
$$

(that is the space of Hopf bimodule morphisms from $B_{q}(M)$ to $C^{p}(N)$ ),

$$
\begin{aligned}
\delta_{h}: C^{p q}(M, N) & \rightarrow C^{p, q+1}(M, N) \\
\alpha & \mapsto \alpha \circ \partial_{q+1}
\end{aligned}
$$

and

$$
\begin{aligned}
\delta_{c}: C^{p q}(M, N) & \rightarrow C^{p+1, q}(M, N) \\
\alpha & \mapsto(-1)^{q} \partial^{p} \circ \alpha .
\end{aligned}
$$

This defines a double complex, and the homology of its total complex is Gerstenhaber and Schack's cohomology of $M$ into $N$; let $H_{G S}^{*}(M, N)$ denote this. 
Remark This double complex is isomorphic to the double complex whose modules are

$$
H o m_{k}\left(A^{\otimes q} \otimes M \otimes A^{\otimes q}, A^{\otimes p} \otimes N \otimes A^{\otimes p}\right),
$$

with differentials $\delta_{h}^{\prime}$ and $\delta_{c}^{\prime}$ defined by:

$$
\begin{aligned}
\delta_{h}^{\prime}(\alpha)\left(\mathbf{a}_{\mathbf{0}, \mathbf{q}} \otimes m \otimes \mathbf{b}_{\mathbf{q}, \mathbf{0}}\right)= & \Delta^{(2 q)}\left(a_{0}\right) \alpha\left(\mathbf{a}_{\mathbf{1}, \mathbf{q}} \otimes m \otimes \mathbf{b}_{\mathbf{q}, \mathbf{1}}\right) \Delta^{(2 q)}\left(b_{0}\right) \\
& +\sum_{i=1}^{q}(-1)^{i} \alpha\left(a_{0} \otimes \ldots \otimes a_{i-1} a_{i} \otimes \ldots \otimes b_{i} b_{i-1} \otimes \ldots\right) \\
& +(-1)^{q+1} \alpha\left(a_{0} \otimes \ldots \otimes a_{q} m b_{q} \otimes \ldots \otimes b_{0}\right)
\end{aligned}
$$

and

$$
\begin{aligned}
(-1)^{q} \delta_{c}^{\prime}(\alpha)= & (1 \otimes \alpha \otimes 1) \circ\left(\delta_{L} \otimes 1\right) \circ \delta_{R}+\sum_{i=1}^{p}(-1)^{i} \tilde{\Delta}_{i} \circ \alpha \\
& +(-1)^{p+1}\left[1^{\otimes p} \otimes\left(\left(1 \otimes \delta_{R}^{(N)}\right) \circ \delta_{L}^{(N)}\right) \otimes 1^{\otimes p}\right] \circ \alpha,
\end{aligned}
$$

where $\tilde{\Delta}_{i}=\Delta_{2 p-i+1} \circ \Delta_{i}$, the notation $\mathbf{a}_{\mathbf{0}, \mathbf{q}}$ stands for $a_{0} \otimes \ldots \otimes a_{q}$, and $\mathbf{b}_{\mathbf{q}, \mathbf{0}}=b_{q} \otimes \ldots \otimes b_{0}$. The various $\delta$ are the coactions on the appropriate Hopf bimodules.

\subsection{Gerstenhaber and Schack cohomology of a Hopf algebra}

When $M$ and $N$ are both equal to the Hopf bimodule $A$, M. Gerstenhaber and S.D. Schack have given, in a similar way, a definition for the cohomology of $A$ (see [GS1]):

Consider the bar resolution $\operatorname{Bar}_{\bullet}(A)$ of $A\left(\operatorname{Bar}_{q}(A)=A^{\otimes q+2}\right)$, and the cobar resolution $\mathrm{Cob}^{\bullet}(A)$ of $A\left(\operatorname{Cob}^{p}(A)=A^{\otimes p+2}\right)$ : they enjoy the same properties as the resolutions $B_{\bullet}(A)$ and $C^{\bullet}(A)$, and the double complex associated to these resolutions is a double complex whose modules are isomorphic to $C^{\prime p q}=\operatorname{Hom}_{k}\left(A^{\otimes q}, A^{\otimes p}\right)$. The homology of its total complex is denoted by $H_{b}^{*}(A, A)$. We shall see further on that $H_{G S}^{*}$ generalizes $H_{b}^{*}$, that is $H_{G S}^{*}(A, A) \cong$ $H_{b}^{*}(A, A)$.

Example When $A$ is a group bialgebra $k G$, it is cosemisimple, so that the rows of the double complex are acyclic, and the homology of the double complex is equal to the homology of the remaining column, that is the cohomology $H^{*}(G, k)=H H^{*}(k G, k)$ (see [GS1]. Further, various cases of group bialgebras have been studied in $[\overline{\mathrm{PW}}]$ ).

Remark There is a suitable sub-double complex $\hat{C}^{\bullet \bullet}$ of $C^{\mathbf{l}^{\bullet \bullet}}$ whose homology is adapted to the study of deformations of the Hopf algebra $A$ as a Hopf algebra. It is obtained from $C^{\prime \bullet \bullet}$ by replacing the first row and the first column by $0 \rightarrow 0 \rightarrow \ldots \rightarrow 0 \rightarrow \ldots$ (see GS1 p79). It has been used by A. Giaquinto in his thesis $([\mathrm{G}])$ to study the preferred deformations of the classical matrix bialgebra and plane (that is deformations in which the comultiplication remains unchanged); he interprets the quantum matrix bialgebra $\mathrm{M}_{q}(2)$ and the quantum plane $\mathrm{k}_{q}^{2}$ as such. 


\subsection{Link with extensions}

In this paragraph, we shall prove that Gerstenhaber and Schack's cohomology $H_{G S}^{*}(M, N)$, and $\operatorname{Ext}_{X}^{*}(M, N)$, are the same. We will first need some definitions and a few preliminary results.

Definition A Hopf bimodule $\mathrm{M}$ is said to be a relative $A-4$ projective when the functor $\operatorname{Hom}_{A 4}(M,-)$ associates exact sequences of vector spaces to exact sequences of Hopf bimodules which split as sequences of $A$-bicomodules. A resolution of a Hopf bimodule is called a relative $A-4$ projective resolution if all its terms are relative $A-4$ projectives and if the sequence splits as a sequence of $A$-bicomodules. One defines dually relative $A$ - 4 injectives and relative $A-4$ injective resolutions (see Sh-St] chapter 10).

Example A projective Hopf bimodule is a relative $A-4$ projective, an injective Hopf bimodule is a relative $A-4$ injective.

Example The resolutions $B \cdot(M)$ and $\operatorname{Bar}_{\bullet}(A)$ are relative $A-4$ projective resolutions; the resolutions $C^{\bullet}(N)$ and $C_{o b} b^{\bullet}(A)$ are relative $A$-4 injective resolutions.

Indeed, let us for instance look at $B \cdot(M)$. In the first place, for any Hopf bimodule $Y$, there is an isomorphism between $\operatorname{Hom}_{A 4}\left(B_{q}(M), Y\right)$ and the space of bicomodule morphisms $H o m^{A A}\left(A^{\otimes q} \otimes M \otimes A^{\otimes q}, Y\right)$, and the functor $\operatorname{Hom}^{A A}\left(A^{\otimes q} \otimes M \otimes A^{\otimes q},-\right)$ clearly takes exact sequences of Hopf bimodules which split as sequences of $A$-bicomodules to exact sequences of vector spaces, so that each $B_{q}(M)$ is a relative $A$-4 projective. Furthermore, we have said earlier that the Bar resolution splits as a sequence of $A$-bicomodules, so that $B \cdot(M)$ is a relative $A-4$ projective resolution of $M$. Similar arguments can be applied to the other resolutions.

These relative resolutions have properties which are similar to those of projective resolutions:

Proposition 3.1 Let $Y$ and $Z$ be two relative $A-4$ projective resolutions of a Hopf bimodule $M$. Then there exists a map of complexes of Hopf bimodules $\phi$ such that the triangle

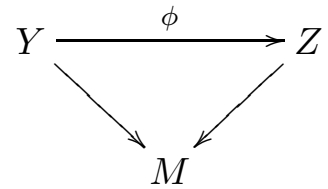

commutes, and $\phi$ is unique up to homotopy of Hopf bimodule complexes. A similar statement is true for relative $A-4$ injective resolutions.

Proof: Follow the proof of the fact that two projective resolutions are homotopic (see [Sh-St] p242-245), bearing in mind that all the exact sequences involved split as sequences of $A$-bicomodules.

Corollary 3.2 Let $Y$ and $T$ be two relative $A-4$ projective resolutions of a Hopf bimodule $M$, and let $Z$ and $U$ be two relative $A-4$ injective resolutions of a Hopf bimodule $N$. Then the double 
complexes $\operatorname{Hom}_{A 4}(Y, Z)$ and $\operatorname{Hom}_{A 4}(T, U)$ endowed with the natural differentials are homotopy equivalent.

Proof: See Sh-St] p246.

Consequences (i) Recall that $B_{\bullet}(M)$ is a relative $A$-4 projective resolution of $M$, therefore in the computation of $H_{G S}^{*}(M, N)$, the resolution $B_{\bullet}(M)$ can be replaced by any relative $A$-4 projective resolution of $M$. The resolution $C^{\bullet}(N)$ can also be replaced by any relative $A$-4 injective resolution of $N$.

(ii) In particular, $H_{G S}^{*}(M, N)$ generalizes $H_{b}^{*}(A, A)$.

We can now prove:

Theorem 3.3 Let $M$ and $N$ be Hopf bimodules over $A$. There is an isomorphism $H_{G S}^{*}(M, N) \cong$ $\operatorname{Ext}_{X}^{*}(M, N)$.

Proof: Let us consider the functor $\operatorname{Ext}_{X}^{*}(-, N)$, with $N$ fixed. It is characterized by the following properties ( $c f$. McD):

1. $\operatorname{Ext}_{X}^{0}(M, N) \cong \operatorname{Hom}_{X}(M, N)=\operatorname{Hom}_{A 4}(M, N)$,

2. $\operatorname{Ext}_{X}^{n}(P, N)=0$ for every $n \geq 1$ and every projective Hopf bimodule $P$,

3. $\operatorname{Ext}_{X}^{*}(-, N)$ is a cohomological $\delta$-functor (see [W] p30).

It is therefore enough to check that the functor $H_{G S}^{*}(-, N)$ satisfies these properties. So the theorem follows from the following three lemmas:

Lemma 3.4 For every Hopf bimodule $M$, there is an isomorphism

$$
H_{G S}^{0}(M, N) \cong \operatorname{Hom}_{A 4}(M, N) .
$$

Let $\alpha$ be an element in $\operatorname{Hom}_{A 4}(A \otimes M \otimes A, A \otimes N \otimes A)$. It is a cocycle if and only if $\delta_{h}(\alpha)=0$ and $\delta_{c}(\alpha)=0$, that is $\alpha \circ \partial_{1}=0=\partial^{1} \circ \alpha$, or $\alpha$ is zero on $\operatorname{Im} \partial_{1}=\operatorname{ker} \partial_{0}$ and $\alpha$ takes values in $\operatorname{ker} \partial^{1}=\operatorname{Im} \partial^{0}$, with $\partial_{0}$ onto and $\partial^{0}$ one-to-one. It follows that if $\alpha$ is a cocycle, it yields a unique morphism $\bar{\alpha} \in \operatorname{Hom}_{A 4}(M, N)$ such that $\partial^{0} \circ \bar{\alpha} \circ \partial_{0}=\alpha$. On the other hand, if $\beta$ is an element of $\operatorname{Hom}_{A 4}(M, N)$, then $\partial^{0} \circ \beta \circ \partial_{0}$ is a cocycle.

Lemma 3.5 For every projective Hopf bimodule $P$ and every integer $n \geq 1$, the $k$-vector space $H_{G S}^{n}(P, N)$ vanishes.

Let $P$ be a projective Hopf bimodule. Then $\cdots 0 \rightarrow 0 \rightarrow \cdots \rightarrow 0 \rightarrow P \rightarrow P \rightarrow 0$ is a relative $A-4$ projective resolution of $P$. It is therefore homotopy equivalent to the bar resolution $B_{\bullet}(M)$ (proposition 3.1). Gerstenhaber and Schack's cohomology is therefore the homology of the double complex in which all the terms are zero except those on the first line, which is equal to $\operatorname{Hom}_{A 4}\left(P, C^{\bullet}(N)\right)$. This line is acyclic, since $P$ is projective and $C^{\bullet}(N)$ is exact. Its cohomology is therefore zero in positive degree. 
Lemma 3.6 The functor $H_{G S}^{*}(-, N)$ takes short exact sequences of Hopf bimodules $0 \rightarrow M^{\prime} \rightarrow$ $M \rightarrow M^{\prime \prime} \rightarrow 0$ to long exact sequences of vector spaces

$$
\cdots \rightarrow H_{G S}^{n}\left(M^{\prime \prime}, N\right) \rightarrow H_{G S}^{n}(M, N) \rightarrow H_{G S}^{n}\left(M^{\prime}, N\right) \stackrel{\delta}{\rightarrow} H_{G S}^{n+1}\left(M^{\prime \prime}, N\right) \rightarrow \cdots
$$

Let $0 \rightarrow M^{\prime} \rightarrow M \rightarrow M^{\prime \prime} \rightarrow 0$ be an exact sequence of Hopf bimodules. Then, for every $q \geq 0$, the sequence

$$
\left(E_{q}\right): 0 \rightarrow A^{\otimes q} \otimes M^{\prime} \otimes A^{\otimes q} \rightarrow A^{\otimes q} \otimes M \otimes A^{\otimes q} \rightarrow A^{\otimes q} \otimes M^{\prime \prime} \otimes A^{\otimes q} \rightarrow 0
$$

is an exact sequence of vector spaces. The sequences

$$
\begin{aligned}
0 \rightarrow \quad & \operatorname{Hom}_{k}\left(A^{\otimes q} \otimes M^{\prime \prime} \otimes A^{\otimes q}, A^{\otimes p} \otimes N \otimes A^{\otimes p}\right) \\
& \rightarrow H o m_{k}\left(A^{\otimes q} \otimes M \otimes A^{\otimes q}, A^{\otimes p} \otimes N \otimes A^{\otimes p}\right) \\
& \rightarrow H o m_{k}\left(A^{\otimes q} \otimes M^{\prime} \otimes A^{\otimes q}, A^{\otimes p} \otimes N \otimes A^{\otimes p}\right) \rightarrow 0
\end{aligned}
$$

are therefore exact for all integers $p, q \geq 0$. These exact sequences commute with the differentials, and thus define an exact sequence of double complexes. This yields a long exact sequence

$$
\cdots \rightarrow H_{G S}^{n}\left(M^{\prime \prime}, N\right) \rightarrow H_{G S}^{n}(M, N) \rightarrow H_{G S}^{n}\left(M^{\prime}, N\right) \stackrel{\delta}{\rightarrow} H_{G S}^{n+1}\left(M^{\prime \prime}, N\right) \rightarrow \cdots
$$

Remark To compute $H_{G S}^{*}(M, N) \cong \operatorname{Ext}_{X}^{*}(M, N)$, one can also take a projective resolution $P_{\bullet}$ of $M$ (or an injective resolution $I^{\bullet}$ of $N$ ) and take the homology of the complex $\operatorname{Hom}_{A 4}\left(P_{\bullet}, N\right)$ (or of the complex $\operatorname{Hom}_{A 4}\left(M, I^{\bullet}\right)$ ).

\section{$4 \quad$ Hopf bimodule cohomology}

When $A$ is a Hopf algebra and $M$ is a Hopf bimodule over $A$, C. Ospel has defined in his thesis [O] a cohomology of $M$ with coefficients in $A$. This can be extended in the following way:

Let $M$ and $N$ be Hopf bimodules. Consider the vector spaces $M \otimes A^{\otimes q+1}$ and $A^{\otimes p+1} \otimes N$ for all non negative integers $p$ and $q$. Each of these vector spaces is endowed with a Hopf bimodule structure:

The space $M \otimes A^{\otimes q+1}$ is equipped with the standard actions and codiagonal coactions, that is

$$
\begin{aligned}
\mu_{L}: A \otimes M \otimes A^{\otimes q+1} & \rightarrow M \otimes A^{\otimes q+1} \\
b \otimes m \otimes \underline{a} & \mapsto b m \otimes \underline{a} \\
\delta_{L}: \quad M \otimes A^{\otimes q+1} & \rightarrow A \otimes M \otimes A^{\otimes q+1} \\
m \otimes \underline{a} & \mapsto \sum m_{(-1)} a_{1}^{(1)} \ldots a_{q+1}^{(1)} \otimes m_{(0)} \otimes a_{1}^{(2)} \otimes \ldots \otimes a_{q+1}^{(2)}
\end{aligned}
$$

and similarly on the right. Dually, $A^{\otimes p+1} \otimes N$ is endowed with the diagonal actions and standard coactions :

$$
\begin{aligned}
\mu_{L}: \quad A \otimes A^{\otimes p+1} \otimes N & \rightarrow A^{\otimes p+1} \otimes N \\
b \otimes \underline{a} \otimes n & \mapsto \sum b^{(1)} a_{1} \otimes \ldots \otimes b^{(p+1)} a_{p+1} \otimes b^{(p+2)} n \\
& =\Delta^{(p+1)}(b)(\underline{a} \otimes n) \\
\delta_{L}: \quad A^{\otimes p+1} \otimes N & \rightarrow A \otimes A^{\otimes p+1} \otimes N \\
\underline{a} \otimes n & \mapsto \sum a_{1}^{(1)} \otimes a_{2}^{(1)} \otimes a_{2} \otimes \ldots \otimes a_{p+1} \otimes n,
\end{aligned}
$$


with similar action and coaction on the right.

For all non negative integers $p$ and $q$, set

$$
K^{p q}(M, N)=\operatorname{Hom}_{A 4}\left(M \otimes A^{\otimes q+1}, A^{\otimes p+1} \otimes N\right),
$$

that is the space of Hopf bimodule morphisms from $M \otimes A^{\otimes q+1}$ to $A^{\otimes p+1} \otimes N$, and consider the maps

$$
\begin{aligned}
d_{h}: K^{p q}(M, N) & \rightarrow K^{p, q+1}(M, N) \\
\alpha & \mapsto \alpha \circ \lambda \\
d_{c}: K^{p q}(M, N) & \rightarrow K^{p+1, q}(M, N) \\
\alpha & \mapsto(-1)^{q} \rho \circ \alpha,
\end{aligned}
$$

where

$$
\begin{array}{rlrl}
\lambda: M \otimes A^{\otimes q+2} & \rightarrow & M \otimes A^{\otimes q+1} \\
m \otimes \underline{a} & \mapsto & m a_{0} \otimes \ldots \otimes a_{q+1} \\
& +\sum_{i=0}^{q}(-1)^{i+1} m \otimes \ldots \otimes a_{i} a_{i+1} \otimes \ldots \otimes a_{q+1}, \text { and } \\
\rho: A^{\otimes p+1} \otimes N & \rightarrow & A^{\otimes p+2} \otimes N \\
x & \mapsto & \left(\sum_{i=0}^{p}(-1)^{i} \Delta_{i}+(-1)^{p+1}\left(1^{\otimes p} \otimes \delta_{L}^{N}\right)\right)(x) .
\end{array}
$$

This defines a double complex. Let $H_{A 4}^{*}(M, N)$ denote the homology of its total complex, which we shall simply call Hopf bimodule cohomology.

Note that $\left(M \otimes A^{\otimes \bullet+1}, \lambda\right)$ is the bar resolution of $M$ as a right module, and $\left(A^{\otimes \bullet+1} \otimes N, \rho\right)$ is the cobar resolution of $N$ as a left comodule.

Remarks (i) This double complex is isomorphic to the double complex whose modules are the spaces of left $A$-module and right $A$-comodule maps

$$
\operatorname{Hom}_{A-}^{-A}\left(M \otimes A^{\otimes q}, A^{\otimes p} \otimes N\right),
$$

the vertical differential translating as $\alpha \mapsto d(\alpha)$ with

$$
\begin{aligned}
d(\alpha)\left(m \otimes a_{1} \otimes \ldots \otimes a_{q+1}\right)= & \alpha\left(m a_{1} \otimes \ldots \otimes a_{q+1}\right) \\
& +\sum_{i=1}^{q}(-1)^{i} \alpha\left(m \otimes \ldots \otimes a_{i} a_{i+1} \otimes \ldots \otimes a_{q+1}\right) \\
& +(-1)^{q+1} \alpha\left(m \otimes a_{1} \otimes \ldots \otimes a_{q}\right) . a_{q+1},
\end{aligned}
$$

and the horizontal differential as

$$
\alpha \mapsto(1 \otimes \alpha) \circ \delta_{L}+\sum_{i=1}^{p}(-1)^{i} \Delta_{i} \circ \alpha+(-1)^{p+1}\left(1^{\otimes p} \otimes \delta_{L}^{N}\right) \circ \alpha .
$$

Furthermore, when $N$ is the Hopf bimodule $A, K^{\bullet \bullet}(M, A)$ is isomorphic to the double complex defined by C. Ospel in his thesis [O].

ii) When $M$ and $N$ are both equal to $A, K^{\bullet \bullet}(A, A)$ is isomorphic to the double complex which defines Gerstenhaber and Schack's bialgebra cohomology $H_{b}^{*}(A, A)$ (see [GS1 and subsection 3.4): $H_{A 4}^{*}(M, N)$ generalizes $H_{b}^{*}(A, A)$. 
(iii) When $N$ is equal to $A$, the homology of the $p^{\text {th }}$ column is the Hochschild cohomology of $A$ with coefficients in $H_{o m}{ }_{A-}\left(M, A^{\otimes p}\right)$, the space of left $A$-module morphisms from $M$ to $A^{\otimes p}$, this last space being endowed with an $A$-bimodule structure as follows: $A^{\otimes p}$ is equipped with the diagonal $A$-bimodule structure, and if $f \in \operatorname{Hom}_{A-}\left(M, A^{\otimes p}\right), a \in A$ and $v \in M$,

$$
(a . f)(v)=f(v \cdot a) \text { and }(f \cdot a)(v)=f(v) a .
$$

The homology of the $q^{t h}$ row is the Cartier cohomology $H_{c}^{*}\left(M \otimes A^{\otimes q}, A\right)$ (see $[\mathrm{C}]$ ).

We shall now study the relationship between this cohomology and the space $\operatorname{Ext}_{X}^{*}(M, N)$.

First, let us state a slight extension of a theorem proved by C. Ospel. We shall also provide a short sketch of the proof, as it will be useful in the proof of theorem 5.3.

Theorem 4.1 (see [0] theorem 3.1) Let $M$ and $N$ be Hopf bimodules. There is an isomorphism

$$
H_{A 4}^{1}(M, N) \cong \operatorname{Ext}_{X}^{1}(M, N) \text {. }
$$

Proof: Take a 1-cochain $f=\left(f_{0}, f_{1}\right)$ in $\operatorname{Hom}_{A-}^{-A}(M \otimes A, N) \oplus \operatorname{Hom}_{A-}^{-A}(M, A \otimes N)$. The corresponding 1-extension $F$ is equal to

$$
0 \rightarrow N \rightarrow N \oplus M \rightarrow M \rightarrow 0
$$

as a sequence of vector spaces, and the $A$-bimodule structure on $N \oplus M$ is given by:

$$
\begin{gathered}
\left(\begin{array}{cc}
\mu_{R}^{N} & f_{0} \\
0 & \mu_{R}^{M}
\end{array}\right) \text { for the right } \mathrm{A} \text { - module structure, } \\
\left(\begin{array}{cc}
\delta_{L}^{N} & f_{1} \\
0 & \delta_{L}^{M}
\end{array}\right) \text { for the left } \mathrm{A} \text { - comodule structure, } \\
\text { and }\left(\begin{array}{cc}
\mu_{L}^{N} & 0 \\
0 & \mu_{L}^{M}
\end{array}\right) \text { and }\left(\begin{array}{cc}
\delta_{R}^{N} & 0 \\
0 & \delta_{R}^{M}
\end{array}\right) \text { for the remaining structures. }
\end{gathered}
$$

The sequence $F$ is a sequence of Hopf bimodules if and only if $f$ is a 1-cocycle. This describes the isomorphism.

More generally, the following is true:

Theorem 4.2 For any Hopf bimodules $M$ and $N$, there is an isomorphism

$$
H_{A 4}^{*}(M, N) \cong \operatorname{Ext}_{X}^{*}(M, N) .
$$

Proof: As in theorem 3.3, we shall prove that the functor $H_{A 4}^{*}(-, N)$ satisfies the properties of the functor $\operatorname{Ext}_{X}^{*}(-, N)$ :

Lemma 4.3 For every Hopf bimodule $M$, there is an isomorphism

$$
H_{A 4}^{0}(M, N) \cong \operatorname{Hom}_{A 4}(M, N) \text {. }
$$

This is proved as in lemma 3.4 . 
Lemma 4.4 For every projective Hopf bimodule $P$ and every integer $n \geq 1$, the $k$-vector space $H_{A 4}^{n}(P, N)$ vanishes.

Let $P$ be a projective Hopf bimodule. Let us consider its bar resolution

$$
B_{\bullet}^{A}(P): \cdots \rightarrow P \otimes A^{\otimes q+2} \stackrel{\lambda_{q}}{\longrightarrow} P \otimes A^{\otimes q+1} \stackrel{\lambda_{q-1}}{\longrightarrow} \cdots \rightarrow P \otimes A^{\otimes 2} \stackrel{\lambda_{0}}{\longrightarrow} P \otimes A \stackrel{\lambda_{-1}}{\longrightarrow} P \rightarrow 0,
$$

with $\lambda_{-1}(u \otimes a)=u . a$.

Since $P$ is projective and $\lambda_{-1}$ is onto, $\lambda_{-1}$ has a section: there exists a morphism of Hopf bimodules $s: P \rightarrow P \otimes A$ satisfying $\lambda_{-1}(s(u))=u$ for every $u \in P$.

Set

$$
\begin{aligned}
h_{q}: P \otimes A^{\otimes q+1} & \longrightarrow P \otimes A^{\otimes q+2} \\
u \otimes a_{0} \otimes \ldots \otimes a_{q} & \mapsto s(u) \otimes a_{0} \otimes \ldots \otimes a_{q} .
\end{aligned}
$$

It is a Hopf bimodule morphism (recall that the Hopf bimodules are endowed with standard actions and codiagonal coactions). Set $s(u)=\sum_{i=1}^{t} v_{i} \otimes b_{i}$, and let us compute $\lambda_{q} h_{q}+$ $h_{q-1} \lambda_{q-1}:$

$$
\begin{aligned}
& \lambda_{q} h_{q}\left(u \otimes a_{0} \otimes \ldots \otimes a_{q}\right)=\lambda_{q}\left(s(u) \otimes a_{0} \otimes \ldots \otimes a_{q}\right) \\
& =\lambda_{q}\left(\sum_{i=1}^{t} v_{i} \otimes b_{i} \otimes a_{0} \otimes \ldots \otimes a_{q}\right) \\
& =\sum_{i=1}^{t}\left[v_{i} \cdot b_{i} \otimes a_{0} \otimes \ldots \otimes a_{q}\right. \\
& -v_{i} \otimes b_{i} a_{0} \otimes a_{1} \otimes \ldots \otimes a_{q} \\
& \left.+\sum_{j=0}^{q-1}(-1)^{j} v_{i} \otimes b_{i} \otimes a_{0} \otimes \ldots \otimes a_{j} a_{j+1} \otimes \ldots \otimes a_{q}\right] \\
& =\lambda_{-1}(s(u)) \otimes a_{0} \otimes \ldots \otimes a_{q} \\
& -\left(s(u) . a_{0}\right) \otimes a_{1} \ldots \otimes a_{q} \\
& +\sum_{j=0}^{q-1}(-1)^{j} s(u) \otimes a_{0} \otimes \ldots \otimes a_{j} a_{j+1} \otimes \ldots \otimes a_{q} \\
& =u \otimes a_{0} \otimes \ldots \otimes a_{q} \\
& -s\left(u . a_{0}\right) \otimes a_{1} \otimes \ldots \otimes a_{q} \\
& +\sum_{j=0}^{q-1}(-1)^{j} s(u) \otimes a_{0} \otimes \ldots \otimes a_{j} a_{j+1} \otimes \ldots \otimes a_{q}
\end{aligned}
$$


and

$$
\begin{aligned}
h_{q-1} \lambda_{q-1}\left(u \otimes a_{0} \otimes \ldots \otimes a_{q}\right)= & h_{q-1}\left(u . a_{0} \otimes a_{1} \otimes \ldots \otimes a_{q}\right) \\
& +\sum_{j=0}^{q-1}(-1)^{j+1} h_{q-1}\left(u \otimes a_{0} \otimes \ldots \otimes a_{j} a_{j+1} \otimes \ldots \otimes a_{q}\right) \\
= & s\left(u . a_{0}\right) \otimes a_{1} \otimes \ldots \otimes a_{q} \\
& -\sum_{j=0}^{q-1}(-1)^{j} s(u) \otimes a_{0} \otimes \ldots \otimes a_{j} a_{j+1} \otimes \ldots \otimes a_{q} .
\end{aligned}
$$

Adding these expressions yields:

$$
\left(\lambda_{q} h_{q}+h_{q-1} \lambda_{q-1}\right)\left(u \otimes a_{0} \otimes \ldots \otimes a_{q}\right)=u \otimes a_{0} \otimes \ldots \otimes a_{q} .
$$

Therefore $h_{\bullet}$ is a Hopf bimodule homotopy from $i d$ to 0 .

Now fix $p \in \mathbb{N}$, and consider the complex $\operatorname{Hom}_{A 4}\left(P \otimes A^{\otimes \bullet+1}, A^{\otimes \bullet+1} \otimes N\right)$; the homotopy $h_{\bullet}$ on $B_{\bullet}^{A}(P)=P \otimes A^{\otimes \bullet+1}$ yields a homotopy $-\circ h_{\bullet}$ from $i d$ to 0 on this complex. Therefore, the double complex:

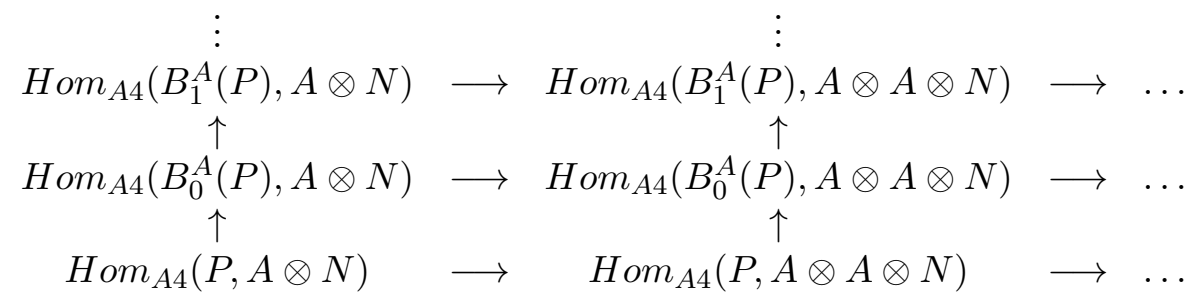

which is the double complex $K^{\bullet \bullet}(P, N)$ to which we have added one row, has exact columns. Its homology is therefore that of the first row $\operatorname{Hom}_{A 4}\left(P, A^{\otimes \bullet+1} \otimes N\right)(c f$. [W] p59-60). Since $P$ is projective and $A^{\otimes \bullet+1} \otimes N$ is exact, the homology of this complex is zero in positive degree.

Lemma 4.5 the functor $H_{A 4}^{*}(-, N)$ takes short exact sequences of Hopf bimodules $0 \rightarrow M^{\prime} \rightarrow$ $M \rightarrow M^{\prime \prime} \rightarrow 0$ to long exact sequences of vector spaces

$$
\cdots \rightarrow H_{A 4}^{n}\left(M^{\prime \prime}, N\right) \rightarrow H_{A 4}^{n}(M, N) \rightarrow H_{A 4}^{n}\left(M^{\prime}, N\right) \stackrel{\delta}{\rightarrow} H_{A 4}^{n+1}\left(M^{\prime \prime}, N\right) \rightarrow \cdots
$$

Consider an exact sequence of Hopf bimodules $0 \rightarrow M^{\prime} \rightarrow M \rightarrow M^{\prime \prime} \rightarrow 0$. Then for all $q \geq 0$, the sequence

$$
\left(E_{q}\right): 0 \rightarrow M^{\prime} \otimes A^{\otimes q} \rightarrow M \otimes A^{\otimes q} \rightarrow M^{\prime \prime} \otimes A^{\otimes q} \rightarrow 0
$$

is also an exact sequence of Hopf bimodules. Now any Hopf bimodule can be written $M \cong$ $A \otimes M^{R}$ where $M^{R}=\left\{m \in M / \delta_{R}(m)=m \otimes 1\right\}$ is the space of right coinvariants of $M$, the left $A$-module and right $A$-comodule structures being given by

$$
\begin{aligned}
b .(a \otimes v) & =b a \otimes v \\
\delta_{R}(a \otimes v) & =\sum a^{(1)} \otimes v \otimes a^{(2)}
\end{aligned}
$$


and therefore any exact sequence of Hopf bimodules splits as a sequence of left $A$-modules and right $A$-comodules, since it yields an exact sequence of coinvariant spaces (a Hopf bimodule is free and therefore injective as a right comodule, and $M^{R} \cong \operatorname{Hom}^{-A}(k, M)$ where $k$ is the trivial right comodule). Thus, the sequences $\left(E_{q}\right)$ split as sequences of left $A$-modules and right $A$-comodules. From this, we infer exact sequences

$$
\begin{aligned}
0 \rightarrow \operatorname{Hom}_{A-}^{-A}\left(M^{\prime \prime} \otimes A^{\otimes q}, A^{\otimes p} \otimes N\right) & \rightarrow \operatorname{Hom}_{A-}^{-A}\left(M \otimes A^{\otimes q}, A^{\otimes p} \otimes N\right) \\
& \rightarrow \operatorname{Hom}_{A-}^{-A}\left(M^{\prime} \otimes A^{\otimes q}, A^{\otimes p} \otimes N\right) \rightarrow 0
\end{aligned}
$$

for all non negative $p$ and $q$. This leads to a long exact sequence

$$
\ldots \rightarrow H_{A 4}^{n}\left(M^{\prime}, N\right) \rightarrow H_{A 4}^{n+1}\left(M^{\prime \prime}, N\right) \rightarrow H_{A 4}^{n+1}(M, N) \rightarrow H_{A 4}^{n+1}\left(M^{\prime}, N\right) \rightarrow \ldots
$$

Remark In fact, we have proved that $H_{A 4}^{*}(M, N)$ and $\operatorname{Ext}_{X}^{*}(M, N)$ are $\delta$-functors which are isomorphic, the isomorphism being the unique map of $\delta$-functors extending the morphism $i d_{H_{\text {om }}(M, N)}$. The same is true with the other variable.

We shall need, in theorem 5.3, the link between theorems 4.1 and 4.2 :

Proposition 4.6 The isomorphism of theorem 4.1 is equal to the isomorphism of theorem 4.2 in degree 1.

Proof: Let us call $\psi_{N}$ the isomorphism $H_{A 4}^{1}(M, N) \sim \operatorname{Ext}_{X}^{1}(M, N)$, and $\varphi_{M N}^{*}$ the isomorphism $H_{A 4}^{*}(M, N) \stackrel{\sim}{\rightarrow} \operatorname{Ext}_{X}^{*}(M, N)$. If we show that $\psi$ commutes with the first connecting homomorphism $\delta^{1}$, then there will be a unique map of $\delta$-functors $H_{A 4}^{*}(M,-) \rightarrow \operatorname{Ext}_{X}^{*}(M,-)$ extending the pair of maps $\left(i d_{H_{o m}(M,-)}, \psi\right)$, which will necessarily be equal to $\varphi_{M}$, since

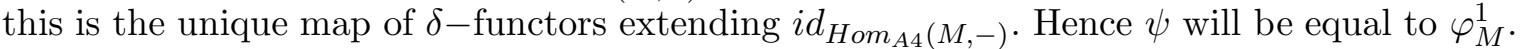

Therefore, let $(E): 0 \rightarrow M^{\prime} \stackrel{i}{\rightarrow} M^{\prime} \oplus M^{\prime \prime} \stackrel{p}{\rightarrow} M^{\prime \prime} \rightarrow 0$ be an exact sequence of Hopf bimodules, where the Hopf bimodule structure of $M^{\prime} \oplus M^{\prime \prime}$ is determined by a 1-cocycle $(\alpha, \beta) \in H_{A 4}^{1}\left(M^{\prime \prime}, M^{\prime}\right)$. Let $g$ be a map in $\operatorname{Hom}_{A 4}(M, N)$. The connecting homomorphism in the long exact sequence associated to $(E)$ for the functor $\operatorname{Ext}_{X}^{*}(M,-)$ is given by Yoneda multiplication on the right by $(E)$, so we want to prove that $\psi_{M^{\prime \prime}}\left(\delta^{1} g\right)=g \circ(E)$ (here $\circ$ is the Yoneda product).

On the one hand, $g \circ(E)$ is the sequence of vector spaces

$$
0 \rightarrow N \rightarrow N \oplus M^{\prime} \oplus M^{\prime \prime} /<\left(g\left(m^{\prime}\right), m^{\prime}, 0\right), m^{\prime} \in M^{\prime}>\rightarrow M^{\prime \prime} \rightarrow 0,
$$

in which the Hopf bimodule stuctures of the middle space are given by

$$
\left(l, m^{\prime}, m^{\prime \prime}\right) \cdot a=\left(l a, m^{\prime} a+\alpha\left(m^{\prime \prime} \otimes a\right), m^{\prime \prime} a\right)=\left(l a+g\left(\alpha\left(m^{\prime \prime} \otimes a\right)\right), m^{\prime} a, m^{\prime \prime} a\right)
$$

so that $\left(l, 0, m^{\prime \prime}\right) \cdot a=\left(l a+g\left(\alpha\left(m^{\prime \prime} \otimes a\right)\right), 0, m^{\prime \prime} a\right)$, and

$$
\begin{aligned}
\delta_{L}\left(l, m^{\prime}, m^{\prime \prime}\right) & =\left(\delta_{L}^{L}(l), \delta_{L}^{M^{\prime}}\left(m^{\prime}\right)+\beta\left(m^{\prime \prime}\right), \delta_{L}^{M^{\prime \prime}}\left(m^{\prime \prime}\right)\right) \\
& =\left(\delta_{L}^{L}(l)+(1 \otimes g) \beta\left(m^{\prime \prime}\right), \delta_{L}^{M^{\prime}}\left(m^{\prime}\right), \delta_{L}^{M^{\prime \prime}}\left(m^{\prime \prime}\right)\right)
\end{aligned}
$$

so $\delta_{L}\left(l, 0, m^{\prime \prime}\right)=\left(\delta_{L}^{L}(l)+(1 \otimes g) \beta\left(m^{\prime \prime}\right), 0, \delta_{L}^{M^{\prime \prime}}\left(m^{\prime \prime}\right)\right)$. 
Thus $g \circ(E)$ is equivalent to $0 \rightarrow L \rightarrow L \oplus M^{\prime \prime} \rightarrow M^{\prime \prime} \rightarrow 0$, in which the Hopf bimodule structure is determined by $(g \alpha,(1 \otimes g) \beta)$.

On the other hand, $\delta^{1} g$ is a 1-cocycle $\left(k_{0}, k_{1}\right) \in H_{A 4}^{1}\left(M^{\prime \prime}, N\right)$ such that $\left(k_{0}(p \otimes 1), k_{1}(p \otimes\right.$ $1))=D h$, with $h \in \operatorname{Hom}_{A-}^{-A}\left(M^{\prime} \oplus M^{\prime \prime}, N\right)$ satisfying $h i=g$. We want to show that $\delta^{1} g$ and $(g \alpha,(1 \otimes g) \beta)$ are equivalent 1-cocycles.

Let us write

$$
\begin{aligned}
h: M^{\prime} \oplus M^{\prime \prime} & \longrightarrow L \\
\left(m^{\prime}, m^{\prime \prime}\right) & \mapsto g\left(m^{\prime}\right)+u\left(m^{\prime \prime}\right) .
\end{aligned}
$$

Then

$$
\begin{aligned}
k_{0}\left(m^{\prime \prime} \otimes a\right) & =h\left(\left(0, m^{\prime \prime}\right) \cdot a\right)-h\left(0, m^{\prime \prime}\right) \cdot a \\
& =h\left(\alpha\left(m^{\prime \prime} \otimes a\right), m^{\prime \prime} a\right)-u\left(m^{\prime \prime}\right) \cdot a \\
& =g \alpha\left(m^{\prime \prime} \otimes a\right)+\delta_{h} u\left(m^{\prime \prime} \otimes a\right) .
\end{aligned}
$$

In the same fashion, we get $k_{1}=(1 \otimes g) \beta+\delta_{c} u$. Therefore $\delta^{1} g=(g \alpha,(1 \otimes g) \beta)+D u$, which provides what we wanted.

Remark The previous results give a unification of various cohomological theories of Hopf bimodules by viewing Hopf bimodules as $X$-modules. There is another way of looking at Hopf bimodules, that is as left modules over the Drinfel'd double $\mathcal{D}(A)$ of $A$. Specifically, there is an equivalence of abelian categories

$$
\begin{array}{ccc}
X-\bmod & \longleftrightarrow & \mathcal{D}(A)-\bmod \\
M & \mapsto & M^{R} \\
A \otimes V & \leftarrow & V
\end{array}
$$

which is exact ( $M^{R}$ is the space of right coinvariants). It follows that

$$
\operatorname{Ext}_{X}^{*}(M, N) \cong \operatorname{Ext}_{\mathcal{D}(A)}^{*}\left(M^{R}, N^{R}\right) .
$$

\section{Cup-product on $H_{A 4}^{*}(M, N)$}

Let $M, N$ and $L$ be Hopf bimodules over $A$. There is a graded product $\operatorname{Ext}_{X}^{*}(M, L) \otimes$ $\operatorname{Ext}_{X}^{*}(L, N) \rightarrow \operatorname{Ext}_{X}^{*}(M, N)$, that is the Yoneda product. Since the space $\operatorname{Ext}_{X}^{*}(M, N)$ is isomorphic to $H_{A 4}^{*}(M, N)$, this is also true for the Hopf bimodule cohomology. In this section, we shall establish a formula for a cup-product

$$
H_{A 4}^{*}(M, L) \otimes H_{A 4}^{*}(L, N) \rightarrow H_{A 4}^{*}(M, N)
$$

(which does not involve $X$ ), and prove that it corresponds to the Yoneda product.

Proposition 5.1 Let $f \in H_{o m_{A-}^{-A}}^{-A}\left(M \otimes A^{\otimes p-s}, A^{\otimes s} \otimes L\right)$ be a $p$-cochain and $g \in \operatorname{Hom}_{A-}^{-A}(L \otimes$ $A^{\otimes q-r}, A^{\otimes r} \otimes N$ ) be a $q$-cochain. Set $n=p+q$ and $t=s+r$. Define the $n$-cochain $f \smile g \in \operatorname{Hom}_{A-}^{-A}\left(M \otimes A^{\otimes n-t}, A^{\otimes t} \otimes N\right)$ by:

$$
\begin{aligned}
& f \smile g\left(m \otimes \mathbf{a}_{\mathbf{1}, \mathbf{n}-\mathbf{t}}\right)= \\
& \quad \sum(-1)^{s(q-r)}\left(1^{\otimes s} \otimes g\right)\left[f\left(m \otimes \mathbf{a}_{\mathbf{1}, \mathbf{p}-\mathbf{s}}\right) \cdot\left(\Delta^{(s-1)}\left(a_{p-s+1}^{(1)} \ldots a_{n-t}^{(1)}\right) \otimes 1\right) \otimes \mathbf{a}_{\mathbf{p}-\mathbf{s}+\mathbf{1}, \mathbf{n}-\mathbf{t}}^{(\mathbf{2})}\right]
\end{aligned}
$$


where $\mathbf{a}_{\mathbf{u}, \mathbf{v}}=a_{u} \otimes \ldots \otimes a_{v}$ and $\mathbf{a}_{\mathbf{u}, \mathbf{v}}^{(\mathbf{2})}=a_{u}^{(2)} \otimes \ldots \otimes a_{v}^{(2)}$.

The differential $D=d_{h}+d_{c}$ of the total complex associated to the Hopf bimodule double complex is a right derivation for the cup-product $\smile$, that is

$$
D(f \smile g)=D f \smile g+(-1)^{p} f \smile D g,
$$

so that the formula for $\smile$ yields a product $H_{A 4}^{*}(M, L) \otimes H_{A 4}^{*}(L, N) \rightarrow H_{A 4}^{*}(M, N)$.

Proof: Some straightforward computations, making use of the properties $\sum \Delta^{(u)}\left(a^{(1)}\right) \otimes$ $\Delta^{(v)}\left(a^{(2)}\right)=\Delta^{(u+v)}(a)$ and $\Delta_{i} \circ \Delta^{(u)}=\Delta^{(u+1)}$, lead to the following formulae:

$$
\begin{aligned}
& d_{h}(f \smile g)=d_{h} f \smile g+(-1)^{p} f \smile d_{h} g \\
& d_{c}(f \smile g)=d_{c} f \smile g+(-1)^{p} f \smile d_{c} g,
\end{aligned}
$$

whence the result.

Remarks (i) When $M$ and $N$ are equal to the Hopf bimodule $A$, the cup-product can be written in the double complex defining $H_{b}^{*}(A, A)$ : take $f$ in $H_{o m}\left(A^{\otimes p-s}, A^{\otimes s}\right)$ and $g$ in $H_{k}\left(A^{\otimes q-r}, A^{\otimes r}\right)$. In this setting, their cup-product becomes

$$
\begin{aligned}
(f \smile g)\left(a_{1} \otimes \ldots \otimes a_{n-t}\right)= & \sum f\left(a_{1}^{(1)} \otimes \ldots \otimes a_{p-s}^{(1)}\right) \Delta^{(s-1)}\left(a_{p-s+1}^{(1)} \ldots a_{n-t}^{(1)}\right) \\
& \otimes \Delta^{(r-1)}\left(a_{1}^{(2)} \ldots a_{p-s}^{(2)}\right) g\left(a_{p-s+1}^{(2)} \otimes \ldots \otimes a_{n-t}^{(2)}\right) .
\end{aligned}
$$

(ii) Note that if $A=k G$ is a group algebra, then $H_{b}^{*}(k G, k G)$ is the Hochschild cohomology $H H^{*}(k G, k)$, and the cup-product on $H_{b}^{*}(k G, k G)$ is the Hochschild cup-product on $H H^{*}(k G, k)$.

Let us give a partial associativity property of this cup-product which will come in useful later:

Proposition 5.2 Let $f, g$ and $h$ be cocycles representing elements in $H_{A 4}^{p}(M, L), H_{A 4}^{q}(L, N)$, and $H_{A 4}^{0}(M, N)$. Then $(f \smile g) \smile h=f \smile(g \smile h)$.

Proof: Indeed, both cocycles are elements of type $\left(u_{t}\right)_{t=0, \ldots, p+q}$ in $\bigoplus_{t=0}^{p+q} \operatorname{Hom}_{A-}^{-A}(M \otimes$ $\left.A^{\otimes p+q-t}, A^{\otimes t} \otimes N\right)$, and in both cases, the degree $t$ component (that is $u_{t}$ ) is equal to

$$
(f \smile g \smile h)_{t}=\left(1^{\otimes t} \otimes h\right) \circ(f \smile g)_{t} .
$$

Let us now establish the link between this cup-product and the Yoneda product of extensions:

Theorem 5.3 Let $M, N$ and $L$ be Hopf bimodules over $A$. Let $\varphi_{M N}: H_{A 4}^{*}(M, N) \rightarrow$ $\operatorname{Ext}_{X}^{*}(M, N)$ be the isomorphism which extends $i d_{H^{\prime} m_{A 4}(M, N)}$, and let

$$
\begin{array}{ccc}
\operatorname{Ext}_{X}^{p}(M, L) \otimes \operatorname{Ext}_{X}^{q}(L, N) & \longrightarrow & \operatorname{Ext}_{X}^{p+q}(M, N) \\
F \otimes G & \mapsto & G \circ F
\end{array}
$$

be the Yoneda product of extensions.

Then, if $f$ is a $p$-cocycle and $g$ is a $q$-cocycle in the Hopf bimodule cohomology, the relationship between the products is given by

$$
\varphi_{M N}^{p+q}(f \smile g)=(-1)^{p q} \varphi_{L N}^{q}(g) \circ \varphi_{M L}^{p}(f):
$$

the cup product and the Yoneda product are equal up to sign. 
Remark If the cup-product is replaced by $f \times g=(-1)^{|f| \cdot|g|} f \smile g$, the product $\times$ is equal to the Yoneda product, but $D$ is then a left derivation for $\times$.

Proof of theorem 5.3: Let $f$ be a cocycle representing an element in $H_{A 4}^{p}(M, L)$, set $F=$ $\varphi_{M L}^{p}(f)$, and consider the maps $\alpha_{N}^{n}=(-1)^{n p} f \smile-: H_{A 4}^{*}(L, N) \rightarrow H_{A 4}^{*}(M, N)$ and the $\operatorname{maps} \beta_{N}^{n}=-\circ F: \operatorname{Ext}_{X}^{n}(L, N) \rightarrow \operatorname{Ext}_{X}^{n+p}(M, N)$ for all $n \in \mathbb{N}$. We need to prove that the diagram

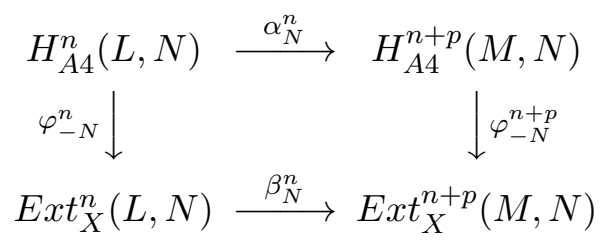

commutes for all $n \in \mathbb{N}$.

First, let us prove that $\alpha$ is a map of $\delta$-functors. Let $0 \rightarrow N^{\prime} \stackrel{u}{\rightarrow} N \stackrel{v}{\rightarrow} N^{\prime \prime} \rightarrow 0$ be an exact sequence of Hopf bimodules; it induces the following diagram:

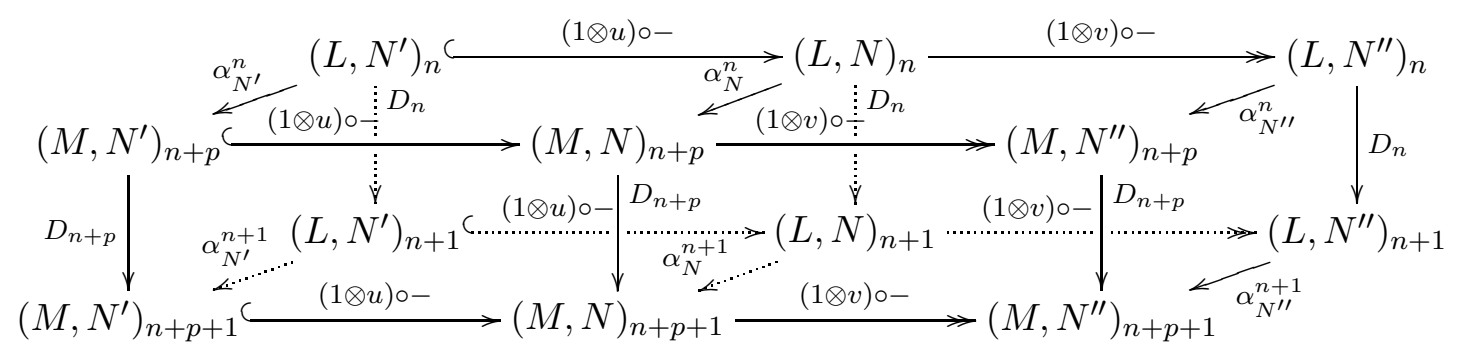

where we have used the notation $(L, N)_{n}:=\bigoplus_{0 \leq r \leq n} H_{o m}^{-A}\left(L \otimes A^{\otimes n-r}, A^{\otimes r} \otimes N\right)$.

By construction of the connecting morphisms, to see that $\alpha$ is a morphism of $\delta$-functors, it is enough to check that all the squares in this diagram commute. The fact that the maps $(1 \otimes u) \circ-$ and $(1 \otimes v) \circ-$ commute with $\alpha$ is easily checked; they also commute with the differential, using the fact that $u$ and $v$ are Hopf bimodule morphisms, and in particular that they are morphisms of right modules and left comodules. Finally, $\alpha$ commutes with the differential, since the latter is a derivation for the cup-product and $f$ is a cocycle. Now the functor $H_{A 4}^{*}(M,-)$ is a universal $\delta$-functor, therefore $\alpha$ is the unique map of $\delta$-functors extending $\alpha^{0}$.

Now consider the maps $\beta_{N}^{n}$. The functor $\operatorname{Ext}_{X}^{*}(M,-)$ is a universal $\delta$-functor, and $\beta$ is a map of $\delta$-functors (indeed, if $E$ is a short exact sequence, the connecting morphism in the cohomological exact sequence it induces for the functor $\operatorname{Ext}_{X}^{*}(L,-)$ is Yoneda multiplication on the right by $E$, see [BK] Chapter 10 Proposition 5). Therefore, $\beta$ is the unique map of $\delta$-functors extending $\beta^{0}$; the map $\beta_{N}^{0}$ is the pushout of $F$ by $g$.

Owing to the universal properties of the maps $\alpha$ and $\beta$, to prove that the diagram (5.1) 
commutes, it is enough to prove that the diagram

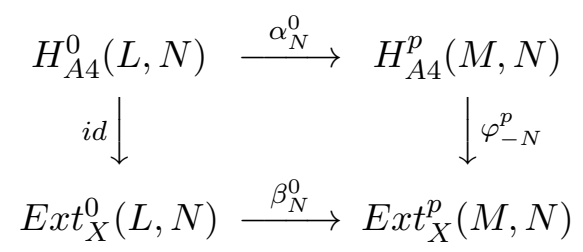

commutes. We shall do it by induction on $p$.

Let us therefore first consider the case where $p=1$. Then $f=\left(f_{0}, f_{1}\right) \in \operatorname{Hom}_{A-}^{-A}(M \otimes$ $A, L) \oplus \operatorname{Hom}_{A-}^{-A}(M, A \otimes L)$ is a 1-cocycle, and $F$ is the exact sequence $0 \rightarrow L \rightarrow L \oplus M \rightarrow$ $M \rightarrow 0$ in which the Hopf bimodule structures are determined by

$$
\left(\begin{array}{cc}
\mu_{R}^{L} & f_{0} \\
0 & \mu_{R}^{M}
\end{array}\right) \text { and }\left(\begin{array}{cc}
\delta_{L}^{L} & f_{1} \\
0 & \delta_{L}^{M}
\end{array}\right)
$$

If $g \in \operatorname{Hom}_{A 4}(M, L)$ is a 0 -cocycle, then $f \smile g$ is a 1-cocycle, and $\varphi_{M N}^{1}(f \smile g)$ is equal to $0 \rightarrow N \rightarrow N \oplus M \rightarrow M \rightarrow 0$ where

$$
\left(\begin{array}{cc}
\mu_{R}^{N} & (f \smile g)_{0} \\
0 & \mu_{R}^{M}
\end{array}\right) \text { and }\left(\begin{array}{cc}
\delta_{L}^{N} & (f \smile g)_{1} \\
0 & \delta_{L}^{M}
\end{array}\right)
$$

determine the Hopf bimodule structures.

Moreover, as in the proof of proposition 4.6, $g \circ F$ is equivalent to the exact sequence $0 \rightarrow N \rightarrow N \oplus M \rightarrow M \rightarrow 0$ in which the Hopf bimodule structures are determined by $g f_{0}$ and $(1 \otimes g) f_{1}$. Since these are respectively equal to $(f \smile g)_{0}$ and $(f \smile g)_{1}$, this proves that $g \circ F$ is equivalent to $\varphi_{M N}^{1}(f \smile g)$. This concludes the first case. It follows that for any $f \in H_{A 4}^{1}(M, L)$ and any $g \in H_{A 4}^{n}(L, N)$,

$$
\varphi_{M N}^{n}(f \smile g)=(-1)^{n} \varphi_{M L}^{n}(g) \circ F .
$$

We can now study the general case: assume $f$ is a $(p+1)$-cocycle. Then we can write $F=E_{p} \circ E_{1}$ where $E_{1}$ is an extension in $\operatorname{Ext}_{X}^{1}(M, R)$ and $E_{p}$ is an extension in $\operatorname{Ext}_{X}^{p}(R, L)$, for a Hopf bimodule $R$. From the first case $(p=1)$, we have

$$
f=\left(\varphi_{M N}^{p+1}\right)^{-1}(F)=\left(\varphi_{M N}^{p+1}\right)^{-1}\left(E_{p} \circ E_{1}\right)=(-1)^{p}\left(\varphi_{M R}^{1}\right)^{-1}\left(E_{1}\right) \smile\left(\varphi_{R L}^{p}\right)^{-1}\left(E_{p}\right) .
$$

Then, if $g$ is a 0 -cocycle in $\operatorname{Hom}_{A 4}(L, N)$, we get

$$
\begin{aligned}
\left(\varphi_{M N}^{p+1}\right)^{-1}(g \circ F) & =\left(\varphi_{M N}^{p+1}\right)^{-1}\left(g \circ E_{p} \circ E_{1}\right) \\
& =(-1)^{p}\left(\varphi_{M R}^{1}\right)^{-1}\left(E_{1}\right) \smile\left(\varphi_{R N}^{p}\right)^{-1}\left(g \circ E_{p}\right) \\
& =(-1)^{p}\left(\varphi_{M R}^{1}\right)^{-1}\left(E_{1}\right) \smile\left[\left(\varphi_{R L}^{p}\right)^{-1}\left(E_{p}\right) \smile\left(\varphi_{L N}^{0}\right)^{-1}(g)\right] \\
& =(-1)^{p}\left[\left(\varphi_{M R}^{1}\right)^{-1}\left(E_{1}\right) \smile\left(\varphi_{R L}^{p}\right)^{-1}\left(E_{p}\right) \smile\left(\varphi_{L N}^{0}\right)^{-1}(g)\right. \\
& =f \smile g
\end{aligned}
$$

using the first case, the induction hypothesis, proposition 5.2 and relation (5.3).

Therefore the diagram (5.2) commutes in general, and this concludes the proof. 


\section{References}

[BKI] Bourbaki, N., Eléments de Mathématiques, Algèbre, Ch.10, Algèbre homologique, Masson (1980).

[CE] Cartan, H. and Eilenberg, S., Homological Algebra, Princeton University Press (1956).

[C] Cartier, P., Cohomologie des Coalgèbres, Sém. Sophus Lie exp.5 (1955-1956).

[CM] Connes, A. and Moscovici, H., Cyclic cohomology and Hopf algebras, Lett. Math. Phys. 48, $\mathrm{n}^{\circ} 1$, pp 97-108 (1999).

[CR] Cibils, C. and Rosso, M., Hopf bimodules are modules, J. Pure and Applied Algebra 128, pp 225-231 (1998).

[GS1] Gerstenhaber, M. and Schack, S.D., Algebras, Bialgebras, Quantum Groups, and Algebraic Deformations, Contemp. Math. 134, pp 51-92 (1992).

[GS2] Gerstenhaber, M. and Schack, S.D., Bialgebra Cohomology, Deformations, and Quantum Groups, Proc. Natl. Acad. Sci. USA 87, pp 478-481 (1990).

[G] Giaquinto, A., Deformation Methods in Quantum Groups, PhD. Thesis, University of Pennsylvania (May 1991).

[McL] MacLane, S., Homology, Springer, New York (1963).

[O] Ospel, C., Thèse, Université Louis Pasteur (Strasbourg I), Strasbourg 1999, Prepublications of the Institute of Advanced Mathematical Research (1999/2).

[PW] Parshall, B. and Wang, J., On Bialgebra Cohomology, Bull. Soc. Math. Belg., Ser. A42, $\mathrm{n}^{\circ} 3, \mathrm{pp} 607-642$ (1990).

[Sh-St] Shnider, S. and Sternberg, S., Quantum Groups - From Coalgebras to Drinfel'd Algebras: A Guided Tour, Cambridge MA International Press Inc. (1993).

[W] Weibel, C., An Introduction to Homological Algebra, Cambridge University Press (1994). 